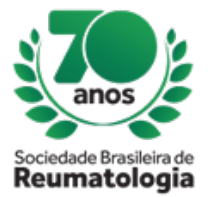

\title{
CUTANEOUS SARCOIDOSIS IN PATIENT WITH RHEUMATOID ARTHRITIS DURING TREATMENT WITH ADALIMUMAB: CASE REPORT
}

Ruy Sampaio Siqueira Neto (Universidade Federal do Ceará, Fortaleza, CE, Brasil), Leila Patrícia Fonseca Oliveira (Universidade Federal do Ceará, Fortaleza, CE, Brasil), André Gois Rocha (Universidade Federal do Ceará, Fortaleza, CE, Brasil), Cristiano Nogueira Marques (Universidade Federal do Ceará, Fortaleza, $\mathrm{CE}$, Brasil), Priscilla Mariana Freitas Aguiar Feitosa (Universidade Federal do Ceará, Fortaleza, CE, Brasil), Mailze Campos Bezerra (Universidade Federal do Ceará, Fortaleza, CE, Brasil)

\section{BACKGROUND}

Sarcoidosis is a multisystemic inflammatory disorder, of unknown etiology, characterized by infiltration of $\mathrm{T}$ lymphocytes mononuclear phagocytes and noncaseating granulomas that can affect several organs or tissues. There is evidence that antagonists of tumor necrosis factor alpha (Anti-TNFla) are useful as treatment. However, disease manifestation has been reported with TNF-a inhibition in other inflammatory conditions. Based on clinical and histopathology findings we report a case of sarcoidosis during treatment with adalimumab.

\section{CASE REPORT}

Patient, female, 62-years-old, rheumatoid arthritis for 33 years. Initially in use of conventional synthetic disease-modifying drugs and prednisone $5 \mathrm{mg} /$ day. Due high disease activity started treatment with adalimumab. Chest $\mathrm{x}$-ray, tuberculin test, and viral serology hepatitis were normal. After 18 months therapy, the patient developed painless subcutaneous nodules, without Inflammatory signs, disseminated in the thighs and buttocks with no other symptom. Adalimumab was suspended and subcutaneous nodule biopsy of the left thigh revealed granulomatous inflammatory infiltrate with granulomas, multinucleated giant cells, with fibrosis and few lymphocytic components. Research for fungi and tuberculosis were negative. Chest x-ray showed no signs of granulomatous disease. After anti-TNFa withdrawal, the lesions disappeared in a few weeks and started abatacept, but the patient persisted in the disease activity. Currently using prednisone and tocilizumab with clinical disease activity index scoring 7 .

\section{CONCLUSION}

The sarcoidosis onset during treatment with adalimumab is rare. In literature there are 64 reported cases of sarcoidosis during Anti-TNFa therapy and only 9 of these have been related to adalimumab. It is postulated that these cases result from an increase reactivity of $T$ lymphocytes and imbalance of cytokines, altering the homeostasis of the immune system responsible for formation and maintenance of the granuloma.

In the reported cases, the appearance of cutaneous lesions ranged from 3 weeks to 60 months. The described lesions were: papular or nodular eruptions, cicatricial lesions and erythema nodosum. In most cases, there was regression after discontinuation of anti-TNF- $\alpha$ therapy in average time of 6 months. A small percentage $(26 \%)$ required low-dose corticosteroids for better response. Our patient evolved with disappearance of the lesions in less than 4 weeks, an early time compared to the cases described in the literature.

The prognosis of sarcoidosis by TNF- $\alpha$ blockade is generally favorable. Some patients may need corticosteroid to decrease inflammatory process. Knowledge of this rare complication is of great importance in view of the increasing use of anti-TNFa in autoimmune diseases and the need to discontinue treatment to improve skin lesions. 